\title{
DMT Bit Rate Maximization With Optimal Time Domain Equalizer Filter Bank Architecture
}

\author{
Milos Milosevic*, Lucio F. C. Pessoa**, Brian L. Evans* ${ }^{1}$ and Ross Baldick* \\ *Dept. of Electrical and Computer Eng., The University of Texas, Austin, TX 78712 \\ **7700 West Parmer Lane MD: TX32-PL30, Motorola, Inc., Austin, TX 78729
}

\begin{abstract}
In a multicarrier modulation system, a time domain equalizer (TEQ) traditionally shortens the transmission channel impulse response (CIR) to mitigate intersymbol interference (ISI). In this paper, we propose a data-rate optimal TEQ filter bank whose data rates at the equalizer output are significantly better than those of the Maximum Bit Rate and Minimum ISI methods and similar to those of the least-squares per-tone equalizer method for ADSL CIRs, transmit filters, and receive filters. The contributions of this paper are: (1) a new model for multicarrier demodulator output SNR that includes ISI, near-end crosstalk, white Gaussian noise, and the digital noise floor; (2) data rate optimal time domain per-tone TEQ filter bank, and (3) a new achievable upper bound on achievable bit rate.
\end{abstract}

\section{Introduction}

Discrete multi-tone (DMT) is a multicarrier modulation method in which the available bandwidth of a communication channel, such as twisted-pair copper media, is divided into numerous subchannels or bins via a fast Fourier transform (FFT). Modulation by the inverse fast Fourier transform (IFFT) and demodulation by FFT create nearly orthogonal subchannels. DMT has been standardized in $[1,2,3,4]$. A similar multicarrier modulation that has been adopted in the Digital Video Broadcasting (DVB) [5] and HYPERLAN-2 [6] standards is called Orthogonal Frequency Division Multiplexing (OFDM).

A guard period called the cyclic prefix (a copy of the last $\nu$ samples of the DMT symbol) is inserted between DMT symbols to reduce inter-carrier interference (ICI) and inter-symbol interference (ISI). A channel impulse response (CIR) longer than $\nu+1$ samples causes both ICI and ISI. In ADSL, $\frac{\nu}{N}=\frac{1}{16}$, where $N$ is the DMT

\footnotetext{
${ }^{1}$ B. Evans was funded by The State of Texas Advanced Technology Program under project 0036580614-2001. E-mail: Lucio.Pessoa@motorola.com, and $\{$ milos, bevans, baldick\}@ece.utexas.edu.
}

symbol length. For downstream transmission, $N=512$ and $\nu=32$. For the upstream, $N=64$ and $\nu=4$.

Time domain equalization eliminates the ISI and ICI from the received DMT signal by shortening the channel impulse response. In a traditional discrete multitone equalizer, the time domain equalization is implemented by a single finite impulse response (FIR) filter, called the time domain equalizer (TEQ). Many TEQ design methods have been published based on the traditional receiver architecture of a single FIR TEQ followed by an FFT and a single-tap FEQ.

Minimum mean-square error (MMSE) is one of the earliest, widely used, and mathematically tractable solutions [7]. The equalizer taps are designed to minimize the mean-square of the residual error between the shortened channel impulse response and a desired target impulse response of length $\nu+1$ samples. MMSE solution does not directly maximize the data rate. MMSE solutions tend to put deep nulls ${ }^{2}$ in the shortened channel impulse response, which in the presence of a digital noise floor (DNF) renders some of the subchannels unable to carry data $[8,9]$.

The Maximum Shortening Signal-to-Noise Ratio (MSSNR) method [10] attempts to maximize the ratio of the energy present in the target shortened channel impulse response window of length $\nu$ samples to the energy outside of the window. The method is based on an observation that reducing the energy of SIR outside the target window will reduce ISI and ICI. MSSNR solution does not directly maximize the data rate. [10].

Maximum Bit Rate (MBR) and Minimum ISI (MinISI) methods [8] are based on a similar idea as [10], which is used to define a new model for the SNR. This SNR model includes ISI and additive white Gaussian noise (AWGN) and is used to determine the bit rate. MBR maximizes the nonlinear bit rate equation by using the Broyden-Fletcher-Goldfarb-Shanno quasiNewton algorithm in Matlab's optimization toolbox.

\footnotetext{
${ }^{2}$ Null in this paper refers to a region of high attenuation in the spectrum of the signal.
} 
The authors in [8] define the Min-ISI method, which minimizes the sum of noise power with the constraint that the power of the signal is constant.

An alternate receiver architecture is proposed in [11]. Since the traditional equalizer equalizes all subchannels "in a combined fashion," which may limit equalization performance, the authors of [11] propose to transfer the TEQ operations to the frequency domain by moving the TEQ into the FEQ. The combined TEQ-FEQ would yield a multi-tap FEQ structure in which each subchannel (tone) is separately equalized.

In this paper, we develop an SNR model based on desire to obtain a circularly convolved data frame and the channel impulse response at the input of the demodulating FFT block and to include the effects of near-end crosstalk (NEXT), AWGN and the DNF. We arrive at the optimal time domain per tone TEQ filter bank (TEQFB) by employing Goertzel filters $[12,13]$ at the receiver during data transmission. We propose a method of assessing performance of TEQ design based on the performance of TEQFB. We show that TEQFB outperforms MBR, Min-ISI and least-squares (LS) PTE achieved bit rates.

The contributions of this paper are: (1) a new model for the signal-to-noise ratio at the FFT output that includes ISI, near-end crosstalk, white Gaussian noise, and the digital noise floor. (2) data rate optimal time domain per-tone TEQ filter bank, and (3) a new achievable upper bound on bit rate performance. ${ }^{3}$

\section{Proposed SNR model}

Let $\mathbf{u}_{i}$ be the $i^{\text {th }} N \times 1$ sample DMT symbol to be decoded. Preceding and following this symbol are $\mathbf{u}_{i-1}$ and $\mathbf{u}_{i+1}$ DMT symbols, respectively. The transmit signal variance is $\sigma_{s}^{2}$. Let $\nu$ be the length of the $\mathrm{CP}$ and $\mathbf{h}=\left[h_{\mathrm{O}}, h_{1}, \cdots, h_{N-1}\right]^{\mathrm{T}}$ be the $N \times 1$ channel impulse response. Let $\mathbf{w}=\left[w_{0}, w_{1}, \cdots, w_{M-1}\right]^{\mathrm{T}}$ be an $M \times 1$ TEQ, where $M$ is some predetermined length. Let $\Delta$ be the transmission delay of the signal from the transmitter to the receiver FFT block and let

$$
\mathbf{U}_{\text {ISI }}^{\Delta}=\mathbf{U}_{i-1}^{\Delta}+\mathbf{U}_{i}^{\Delta}+\mathbf{U}_{i+1}^{\Delta}
$$

be the convolutional matrix of the DMT symbols $i-$ $1, i$ and $i+1$. Define the $N \times(N+M-1)$ matrix $\mathbf{U}_{i}^{\Delta}=\left[\left(\mathbf{U}_{i}^{\Delta}\right)_{L}\left(\mathbf{U}_{i}^{\Delta}\right)_{R}\right]$, where $\left(\mathbf{U}_{i}^{\Delta}\right)_{R}$ and $\left(\mathbf{U}_{i}^{\Delta}\right)_{L}$

\footnotetext{
${ }^{3} \mathrm{~A}$ more complete analysis of the proposed TEQ design can be found [14], which also the single TEQ case.
}

are respectively given by

$$
\left[\begin{array}{llllll}
0 & \cdots & \cdots & \cdots & \cdots & 0 \\
u_{i}^{N-\nu} & \cdots & \cdots & \cdots & \cdots & 0 \\
\vdots & & \vdots & \vdots & & \vdots \\
u_{i}^{N-2} & \cdots & u_{i}^{N-\nu} & 0 & \cdots & 0 \\
u_{i}^{N-1} & \cdots & u_{i}^{N-\nu-1} & 0 & \cdots & 0 \\
\vdots & & \vdots & \vdots & & \vdots \\
u_{i}^{N-2} & \cdots & u_{i}^{0} & u_{i}^{N-1} & \cdots & u_{i}^{N-\nu} \\
\vdots & & \vdots & \vdots & & \vdots \\
0 & \cdots & 0 & u_{i}^{N-1} & \cdots & u_{i}^{\Delta-M-1}
\end{array}\right]
$$

$$
\left[\begin{array}{llllll}
u_{i}^{\Delta} & \cdots & u_{i}^{0} & u_{i}^{N-1} & \cdots & u_{i}^{N-\nu} \\
u_{i}^{\Delta+1} & \cdots & \cdots & u_{i}^{0} & u_{i}^{N-1} & \cdots \\
\vdots & & & & \vdots & \vdots \\
u_{i}^{N-1} & \cdots & \cdots & \cdots & u_{i}^{0} & u_{i}^{N-1} \\
0 & u_{i}^{N-1} & \cdots & \cdots & u_{i}^{1} & u_{i}^{0} \\
\vdots & & & & \vdots & \vdots \\
0 & \cdots & \cdots & \cdots & 0 & u_{i}^{N-1} \\
\vdots & & & & \vdots & \vdots \\
0 & \cdots & \cdots & \cdots & 0 & 0
\end{array}\right]
$$

Matrices $\mathbf{U}_{i+1}^{\Delta}$ and $\mathbf{U}_{i-1}^{\Delta}$ of size $N \times(N+M-1)$ are respectively given by

$$
\left[\begin{array}{ccccccc}
- & & & \mathbf{0} & & & \\
u_{i+1}^{N-\nu} & 0 & \cdots & \cdots & 0 & \cdots & 0 \\
\vdots & & & & & & \vdots \\
u_{i+1}^{0} & u_{i+1}^{N-1} & \ldots & u_{i+1}^{N-\nu} & 0 & \cdots & 0 \\
\vdots & \ddots & \ddots & & \ddots & \ddots & \vdots \\
u_{i+1}^{\Delta-\nu-1} & \cdots & u_{i+1}^{0} & u_{i+1}^{N-1} & \cdots & u_{i+1}^{N-\nu} & 0
\end{array}\right]
$$

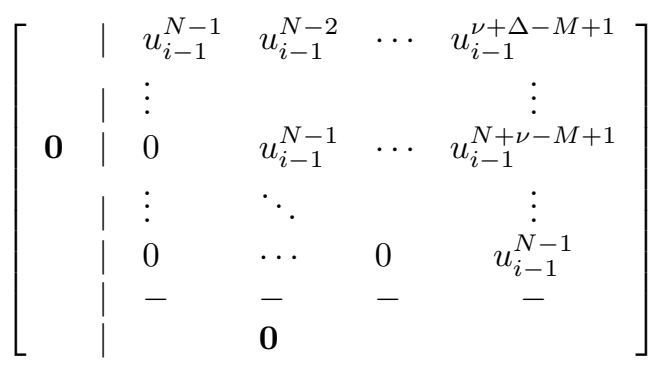


Let $\mathbf{H}$ be the $(N+M-1) \times M$ convolution matrix of the CIR and TEQ given by

$$
\left[\begin{array}{llllc}
h_{0} & 0 & 0 & \cdots & 0 \\
h_{1} & h_{0} & 0 & \cdots & 0 \\
\vdots & & & & \vdots \\
h_{M-1} & \cdots & & \cdots & h_{0} \\
\vdots & & & & \vdots \\
h_{N-1} & \cdots & & \cdots & h_{N-1-M} \\
0 & h_{N-1} & \cdots & \cdots & h_{N-M} \\
\vdots & & & & \vdots \\
0 & 0 & 0 & \cdots & h_{N-1}
\end{array}\right]
$$

We define the vector

$$
\mathbf{q}_{k}=\left[1, e^{j 2 \pi k / N}, \cdots, e^{j 2 \pi(N-1) k / N}\right]^{\mathrm{T}}
$$

such that the inner product of $\mathbf{q}_{k}^{\mathrm{H}}$ with an $N$-point vector gives the $k^{\text {th }}$ FFT coefficient of that vector, where $(\cdot)^{\mathrm{H}}$ is the Hermitian (conjugate transpose) operator.

Let the NEXT and AWGN noise vectors be

$$
\mathbf{n}^{\mathrm{xx}}=\left\{n_{-M+1}^{\mathrm{xx}}, n_{-M+2}^{\mathrm{xx}}, \cdots, n_{0}^{\mathrm{xx}}, \cdots, n_{N-1}^{\mathrm{xx}}\right\}^{\mathrm{T}}
$$

where the subscript $(.)_{\mathrm{xx}}$ is equal to either NEXT or AWGN. Then the $(N+M-1) \times M$ AWGN and NEXT convolution matrices with the TEQ, $\mathbf{G}_{\text {AWGN }}$ and $\mathbf{G}_{\mathrm{NEXT}}$ are

$$
\mathbf{G}_{\mathrm{xx}}=\left[\begin{array}{cccc}
n_{0}^{\mathrm{xx}} & n_{-1}^{\mathrm{xx}} & \cdots & n_{-M+1}^{\mathrm{xx}} \\
n_{1}^{\mathrm{xx}} & n_{0}^{\mathrm{xx}} & \cdots & n_{-M+2}^{\mathrm{xx}} \\
\vdots & & & \vdots \\
n_{M-1}^{\mathrm{xx}} & \cdots & \cdots & n_{0}^{\mathrm{xx}} \\
\vdots & & & \vdots \\
n_{N-1}^{\mathrm{xx}} & \cdots & & n_{N-1-M}^{\mathrm{xx}} \\
\vdots & & & \vdots \\
n_{N+M}^{\mathrm{xx}} & n_{N+M-1}^{\mathrm{xx}} & \cdots & n_{N-1}^{\mathrm{xx}}
\end{array}\right]
$$

The samples of AWGN are i.i.d. according to $\mathcal{N}\left(0, \sigma_{\text {AWGN }}^{2}\right)$ where $\sigma_{\text {AWGN }}^{2}$ is the noise variance, which is measured by the power of the noise with respect to $100 \Omega$ resistance. Samples of the NEXT have spectrally shaped power spectral density (PSD) as defined in [1] for various types of interferers and denoted using the Toeplitz variance matrix $\boldsymbol{\Sigma}_{\text {NEXT }}$.

Define $D_{k}$ as the DNF term in the subchannel $k$ due to noise induced by digital signal processing, which is independent of the TEQ but affects the subchannel $\mathrm{SNR}$ terms, $\mathrm{SNR}_{k}(\mathbf{w})$. The DNF are i.i.d. according to $\mathcal{U}\left(0, \sigma_{\text {DNF }}^{2}\right)$, where $\mathcal{U}$ stands for uniform distribution and $\sigma_{\mathrm{DNF}}^{2}=2^{-2(p+1)} / 3$, where $p$ is the number of bits of numerical precision.
Using these definitions, we write the received data point in the $k^{\text {th }}$ subchannel as

$$
Y_{\mathrm{R}}^{k}(\mathbf{w})=\mathbf{q}_{k}^{\mathrm{H}}\left(\mathbf{U}_{\mathrm{ISI}}^{\Delta} \mathbf{H}+\mathbf{G}_{\mathrm{AWGN}}+\mathbf{G}_{\mathrm{NEXT}}\right) \mathbf{w}+D_{k}
$$

where $k \in\left\{0, \cdots, \frac{N}{2}-1\right\}$. The received data contains the noise due to the ISI, ICI, AWGN, NEXT and DNF, and suffers from the effects of the channel. Now, we see the dependence of the received signal on the TEQ. The ideal received signal has no noise present and is "formatted" to fit the demodulation scheme. In the case of DMT, this means that the received symbol has minimal noise present due to AWGN, NEXT, and ISI and that the strength of the signal is much higher than the DNF. We can design the TEQ to process the received samples to optimize these goals.

Next, we will express the desired signal as a function of the TEQ taps. Ideally, after the TEQ, one would prefer to have mimicked a circular convolution of the signal and the channel impulse response using the cyclic prefix. The desired circular convolution of the $i^{\text {th }}$ symbol and the CIR in the $k^{\text {th }}$ subchannel, after the TEQ and FFT, can be written as

$$
Y_{\mathrm{D}}^{k}(\mathbf{w})=\mathbf{q}_{k}^{\mathrm{H}}\left[\mathbf{U}_{i}^{\Delta}\right]_{\text {circ }} \mathbf{H w}, k \in\{0, \cdots, N / 2-1\}
$$

and the $N \times(N+M-1)$ circulant matrix $\left[\mathbf{U}_{i}^{\Delta}\right]_{\text {circ }}$ is

$$
\left[\begin{array}{lllllc}
u_{i}^{\Delta} & \cdots & u_{i}^{0} & u_{i}^{N-1} & \cdots & u_{i}^{\Delta-M+2} \\
\vdots & & & & \vdots & \\
u_{i}^{N-1} & \cdots & u_{i}^{\Delta} & u_{i}^{\Delta-1} & \cdots & u_{i}^{N-M+1} \\
u_{i}^{0} & \cdots & u_{i}^{\Delta+1} & u_{i}^{\Delta} & \cdots & u_{i}^{N-M+2} \\
\vdots & & & & & \vdots \\
u_{i}^{\Delta-1} & \cdots & u_{i}^{0} & u_{i}^{N-1} & \cdots & u_{i}^{\Delta-M-1}
\end{array}\right]
$$

So, now the received data $Y_{\mathrm{R}}^{k}(\mathbf{w})$ can be rewritten as

$$
Y_{\mathrm{R}}^{k}(\mathbf{w})=Y_{\mathrm{D}}^{k}(\mathbf{w})+\underbrace{\left(Y_{\mathrm{R}}^{k}(\mathbf{w})-Y_{\mathrm{D}}^{k}(\mathbf{w})\right)}_{\text {ISI, ICI, AWGN, NEXT, DNF }}
$$

We then write $\mathrm{SNR}^{\mathrm{Model}}{ }_{k}(\mathbf{w})$ for all $k$ as

$$
\frac{E\left\{\left[Y_{\mathrm{D}}^{k}(\mathbf{w})\right]^{H} Y_{\mathrm{D}}^{k}(\mathbf{w})\right\}}{E\left\{\left[Y_{\mathrm{R}}^{k}(\mathbf{w})-Y_{\mathrm{D}}^{k}(\mathbf{w})\right]^{\mathrm{H}}\left[Y_{\mathrm{R}}^{k}(\mathbf{w})-Y_{\mathrm{D}}^{k}(\mathbf{w})\right]\right\}}
$$

where $E[\cdot]$ is the statistical expectation operator and $[\cdot]^{\text {Model }}$ stands for model. The proposed SNR model is the ratio of the desired data, which excludes the effects of the noise including the ISI and ICI, to the difference between the received data and the desired data.

With some algebra we arrive at

$$
\begin{aligned}
E\left[\left(Y_{\mathrm{D}}^{k}\right)^{\mathrm{H}} Y_{\mathrm{D}}^{k}\right] & =\sigma_{s}^{2} \mathbf{w}^{\mathrm{T}} \mathbf{H}^{\mathrm{T}} \mathbf{Q}_{k}^{\text {circ }}\left[\mathbf{Q}_{k}^{\text {circ }}\right]^{\mathrm{H}} \mathbf{H} \mathbf{w} \\
& =\mathbf{w}^{\mathrm{T}} \tilde{\mathbf{A}}_{k} \mathbf{w}
\end{aligned}
$$


where $\mathbf{Q}_{k}^{\text {circ }}$ is an $(N+M-1) \times N$ matrix

$$
\left[\begin{array}{ccccc}
q_{k}^{N D+1} & \cdots & q_{k}^{N 1} & \cdots & q_{k}^{N D} \\
q_{k}^{N D+2} & \cdots & q_{0} & \cdots & q_{k}^{N D+1} \\
\vdots & & & & \vdots \\
q_{k}^{0} & \cdots & \cdots & \cdots & q_{k}^{N 1} \\
\vdots & & & & \vdots \\
q_{k}^{M D-3} & \cdots & q_{k}^{0} & \cdots & q_{k}^{N-M D-2}
\end{array}\right]
$$

and $N D=N-\Delta, M D=M+\Delta$ and $N 1=N-1$. Note that if the power allocated to subchannels is not equal in all subchannels we would need to include a diagonal matrix that would contain these different powers on the diagonal instead of just $\sigma_{s}^{2}$.

Similarly, we derive the denominator of (14) to be equal to $\mathbf{w}^{\mathrm{T}} \tilde{\mathbf{B}}_{k} \mathbf{w}$ where

$$
\begin{aligned}
\tilde{\mathbf{B}}_{k} & =2 \sigma_{s}^{2}\left(\mathbf{H}_{\mathrm{u}}^{\mathrm{T}} \mathbf{V}_{k} \mathbf{V}_{k}^{\mathrm{H}} \mathbf{H}_{\mathrm{u}}+\mathbf{H}_{\mathrm{b}}^{\mathrm{T}} \mathbf{W}_{k} \mathbf{W}_{k}^{\mathrm{H}} \mathbf{H}_{\mathrm{b}}\right) \\
& +\sigma_{\text {AWGN }}^{2} \mathbf{Q}_{k}^{\text {noise }}\left[\mathbf{Q}_{k}^{\text {noise }}\right]^{\mathrm{H}} \\
& +\mathbf{Q}_{k}^{\text {noise }} \boldsymbol{\Sigma}_{\mathrm{NEXT}}\left[\mathbf{Q}_{k}^{\text {noise }}\right]^{\mathrm{H}}+\frac{\sigma_{\text {DNF }}^{2} \mathbf{I}}{\mathbf{w}^{\mathrm{T}} \mathbf{W}}
\end{aligned}
$$

and $\mathbf{I}$ is the $M \times M$ identity matrix. Without loss of generality, define constraint set $\mathcal{S}=\left\{\mathbf{w}: \mathbf{w}^{\mathrm{T}} \mathbf{w}=1\right\}$, so that $\tilde{\mathbf{B}}_{k}$ becomes independent of TEQ FIR taps $\mathbf{w}$. Matrix $\mathbf{Q}_{k}^{\text {noise }}$ of size $M \times(N+M-1)$ is defined as

$$
\left[\begin{array}{ccccccc}
0 & 0 & \cdots & 0 & q_{k}^{0} & \cdots & q_{k}^{N-1} \\
0 & 0 & \cdots & q_{k}^{0} & \cdots & q_{k}^{N-1} & 0 \\
\vdots & & & & & & \vdots \\
q_{k}^{0} & \cdots & q_{k}^{N-1} & 0 & \cdots & 0 & 0
\end{array}\right]
$$

where $q_{k}^{(\cdot)}$ are members of the vector $\mathbf{q}_{k}(7) . \mathbf{V}_{k}$ is a $\Delta \times \Delta$ upper diagonal matrix defined as

$$
\left[\begin{array}{llllc}
q_{k}^{N-\Delta} & q_{k}^{N-\Delta+1} & \cdots & q_{k}^{N-2} & q_{k}^{N-1} \\
q_{k}^{N-\Delta+1} & q_{k}^{N-\Delta} & \cdots & q_{k}^{N-1} & 0 \\
\vdots & & & \vdots & \vdots \\
q_{k}^{N-1} & 0 & \cdots & 0 & 0
\end{array}\right]
$$

$\mathbf{W}_{k}$ is a lower diagonal $(N-\nu-\Delta+M-1) \times(N-$ $\nu-\Delta+M-1)$ matrix defined as

$$
\left[\begin{array}{ccclc}
0 & 0 & \cdots & 0 & q_{k}^{0} \\
0 & 0 & \cdots & q_{k}^{0} & q_{k}^{1} \\
\vdots & & & \vdots & \vdots \\
q_{k}^{0} & q_{k}^{1} & \cdots & \cdots & q_{k}^{N-\nu-\Delta+M-2}
\end{array}\right]
$$

and

$$
\mathbf{H}=\left[\begin{array}{l}
\left(\mathbf{H}_{\mathrm{u}}\right)_{\Delta \times M} \\
\left(\mathbf{H}_{\mathrm{c}}\right)_{\nu \times M} \\
\left(\mathbf{H}_{\mathrm{b}}\right)_{(N-\nu-\Delta+M-1) \times M}
\end{array}\right]
$$

$\tilde{\mathbf{A}}_{k}$ and $\tilde{\mathbf{B}}_{k}$ are Hermitian symmetric. The SNR model (14) is

$$
\operatorname{SNR}_{k}^{\text {Model }}(\mathbf{w})=\frac{\mathbf{w}^{\mathrm{T}} \tilde{\mathbf{A}}_{k} \mathbf{w}}{\mathbf{w}^{\mathrm{T}} \tilde{\mathbf{B}}_{k} \mathbf{w}}
$$

$\mathrm{SNR}_{k}^{\mathrm{Model}}(\mathbf{w})$ is a ratio of quadratic functions of $\mathbf{w}$. A similar SNR form has been derived in [8]. The SNR model becomes equivalent to the SNR that could be measured at the output of the FFT in an ADSL system when the ISI and ICI have been removed from the received signal.

\section{Optimal TEQ Filter Bank Design}

There are $N / 2$ possible data carrying subchannels in DMT. We propose a method of finding the optimal w for every one of them. Using the SNR model, the number of bits per symbol that can be supported is

$$
\begin{aligned}
b_{\mathrm{DMT}}(\mathbf{w}) & =\sum_{k \in \mathcal{I}} \log _{2}\left(1+\frac{\mathrm{SNR}_{k}^{\mathrm{Model}}(\mathbf{w})}{\Gamma}\right) \\
& =\sum_{k \in \mathcal{I}} \underbrace{\log _{2}\left(\frac{\mathbf{w}^{\mathrm{T}} \mathbf{A}_{k} \mathbf{w}}{\mathbf{w}^{\mathrm{T}} \mathbf{B}_{k} \mathbf{w}}\right)}_{b_{k}(\mathbf{w})}
\end{aligned}
$$

where $\Gamma$ is the SNR gap $[15,16], \mathcal{I}$ is a set of datacarrying subchannels, $\mathbf{A}_{k}=\Gamma \tilde{\mathbf{B}}_{k}+\tilde{\mathbf{A}}_{k}$ and $\mathbf{B}_{k}=\Gamma \tilde{\mathbf{B}}_{k}$, and $b_{k}(\mathbf{w})$ is the number of bits per data symbol in the subchannel $k$.

Maximizing the number of bits allocated in a single channel, $b_{k}(\mathbf{w})$, involves maximizing the argument of the log function, which is a single quadratic ratio. This is the well-known generalized eigenvalue problem [17] and the solution is the generalized eigenvector $\mathbf{w}_{k}^{\text {opt }}$ corresponding to the largest generalized eigenvalue $\lambda_{k}^{\text {opt }}$ of $\left(\mathbf{A}_{k}^{r}, \mathbf{B}_{k}^{r}\right)$ where $(.)^{r}$ denotes the real part.

The number of bits per symbol with the optimal per-tone TEQ filter bank (TEQFB) with $|\mathcal{I}|$ TEQs is

$$
b_{\mathrm{DMT}}^{\mathrm{opt}}=\sum_{k \in \mathcal{I}} \log _{2}\left[\frac{\left(\mathbf{w}_{k}^{\mathrm{opt}}\right)^{\mathrm{T}} \mathbf{A}_{k} \mathbf{w}_{k}^{\mathrm{opt}}}{\left(\mathbf{w}_{k}^{\mathrm{opt}}\right)^{\mathrm{T}} \mathbf{B}_{k} \mathbf{w}_{k}^{\mathrm{opt}}}\right]
$$

The FFT block can be implemented as a bank of Goertzel filters [12] with each one computing a single point DFT. Data transmission computational complexity is higher than with the single TEQ. The equation (24) defines the upper bound on the achievable data rate.

The above discussion assumed that the TEQ subchannel filters are of equal size $M$ for simplicity, however the member filters of TEQFB can have different sizes. In such TEQFB variant, the ISI-heavy subchannels may be given a longer subchannel TEQ, whereas the subchannels with low ISI content may be given 
shorter subchannel TEQs. This approach is very expensive and not practical as the search area spans all subchannels and various sizes of TEQ filters.

\section{Simulation Results}

The simulation results compare the performance of the proposed TEQ design method with Min-ISI, MBR and LS PTE initialized using the least-squares method. We use the eight standard downstream CSA loops [1] convolved with transmit and receive filters as the test CIR. The transmit and receive filters are modelled as firstorder high-pass IIR filters, which are designed to separate ADSL from the $0-4 \mathrm{kHz}$ voice band (a double zero is located at $z=1$, while conjugate symmetric poles are located at $z=0.9799 \pm \mathrm{j} 0.0317)$. All CIRs consist of 512 samples sampled at $2.208 \mathrm{MHz}$. We use the FFT size $N=512$ standard in downstream ADSL with $\Gamma=9.8 \mathrm{~dB}$, thus we do not add the effects of possible coding gain or margin All of the powers used in the simulations are defined with respect to a $100 \Omega$ resistance. The power of the signal is $0.2475 \mathrm{~W}$ spread equally over all of the subchannels. AWGN power is equal to -140 $\mathrm{dBm} / \mathrm{Hz}$ over the bandwidth of $1.104 \mathrm{MHz}$ with the NEXT source being modelled as 49 ADSL disturbers. The PSD of the NEXT is defined in the ADSL standard [1]. Delay $\Delta$ is taken as a free parameter in the optimization and is varied over values 0 to 40 with the best achieved data rate taken to be the best result. The upper limit of 40 was heuristically determined for the tested channels. Thus, once $\Delta_{\text {opt }}$ is chosen, the simulation software proceeds with the determination of the TEQ block according to the method in question (TEQFB, MBR, etc.)

TEQ FIR designed by Min-ISI and MBR methods is obtained using the Matlab DMT TEQ Design Toolbox [18]. LS PTE structures of [11] were designed using the least-squares method that does not require knowledge of the channel model. The same number of symbols (300) were used to let the LS PTE converge for all TEQ sizes $M$.

We measure the SNR in our simulations as it would be measured in a real ADSL DMT system during modem initialization. During training, all subchannels are loaded with a randomly chosen two-bit constellation point at the transmitter. QAM decoding at the receiver compares the complex value received in a subchannel with the transmitted value so that SNR measurement can be derived from the power of the error averaged over 1000 symbols for any particular subchannel $k$. The SNR measurement equation is

$$
\mathrm{SNR}_{k}=10 \log _{10}\left(\frac{2}{\frac{1}{S} \sum_{i=0}^{S-1}\left\|X_{k}^{i}-Y_{k}^{i}\right\|^{2}}\right)
$$

Table 1: Highest achieved bit rates in Mbps of the proposed TEQFB for the CIR involving standard CSA loops 1-8 with transmit and receive high-pass filters for $N=512$ and $\nu=32$ with input power $=\mathbf{0 . 2 4 7 2}$ $W, A W G N$ power $=-140 \mathrm{dBm} / \mathrm{Hz}$, NEXT modelled as 49 ADSL disturbers and optimal $\triangle$. Each TEQFB subchannel TEQ filter has $M$ taps. Beyond $M$ taps, the data rate exhibits diminishing returns.

\begin{tabular}{|c||c||c||c|}
\hline CIR of & TEQFB (Mbps) & $\boldsymbol{\Delta}_{\text {opt }}$ & $M$ \\
\hline \hline CSA loop 1 & 11.417 & 15 & 8 \\
\hline CSA loop 2 & 12.680 & 22 & 12 \\
\hline CSA loop 3 & 10.995 & 26 & 8 \\
\hline CSA loop 4 & 11.288 & 35 & 6 \\
\hline CSA loop 5 & 11.470 & 32 & 16 \\
\hline CSA loop 6 & 10.861 & 20 & 8 \\
\hline CSA loop 7 & 10.752 & 34 & 13 \\
\hline CSA loop 8 & 9.615 & 35 & 11 \\
\hline
\end{tabular}

where $S=1000$ symbols, $X_{k}^{i}$ is the transmitted 2bit constellation data in the subchannel $k$ in the $i^{\text {th }}$ symbol, $Y_{k}^{i}$ is the received data in the subchannel $k$ in the $i^{\text {th }}$ symbol and 2 comes from the power of two-bit constellation point at locations $( \pm 1, \pm 1)$. The bit rates reported are calculated using the measured SNR (25) on subchannels 7-256, following the ADSL standard for $10^{-7}$ BER and up to 15 bits allowed per subchannel.

Proposed TEQFB, Min-ISI, MBR and LS PTE design methods are evaluated using this SNR measurement, which establishes a common testing platform. In Fig. 1 we show how the achieved bit rate varies with the change in the number of TEQ taps $M$ ranging from 2 to 32 for the CIRs containing CSA loop 2, 3 and 7. The bit rate grows significantly from 2 to 3 TEQ taps. The upward slope is present with the further increase in the number of TEQ taps $M$ but it is significantly moderated.

Table 1 lists the data rate achieved with the proposed optimal TEQFB for the CIR including CSA loops 1-8. These data rates represent the maximum data rate that can be achieved as a function of TEQFB for the given signal and noise power levels.

\section{Acknowledgements}

The authors would like to thank Mr. Ketan J. Mandke and Ms. Esther I. Resendiz of The University of Texas (Austin, TX) for their Matlab implementations of part of the proposed TEQFB design algorithm. 

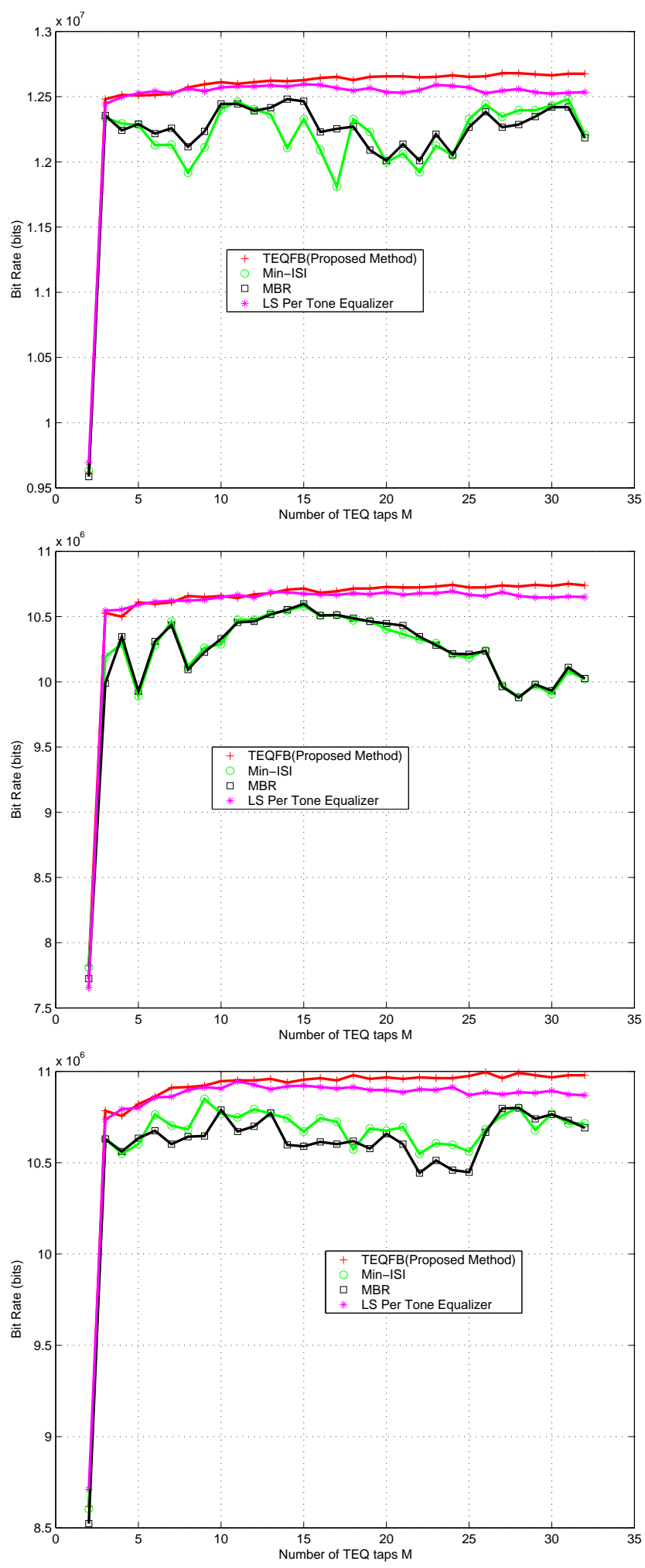

Figure 1: Data rates achieved for different number of TEQ taps $M$ for the CIR containing CSA loop 2 (top), 7 (center) and 3 (bottom) for $N=512$ and $\nu=32$ with input power $=0.2472 \mathrm{~W}$, AWGN power $=-140 \mathrm{dBm} / \mathrm{Hz}$, NEXT modelled as $49 \mathrm{ADSL}$ disturbers. Note that the reported rates are up to $\pm 60 \mathrm{Kbps}$ away from true values.

\section{References}

[1] ANSI, "Network and customer installation interfaces: Asymmetric digital subscriber line (ADSL) metallic interface," in American National Standard for Telecomm., no. T1E1.413, 1998.

[2] ITU-T, "Asymmetrical digital subscriber line (ADSL) transceivers," in Int. Telecomm. Union, no. G.992.1, 1999.

[3] ITU-T, "Splitterless asymmetric digital subscriber line (ADSL) transceivers," in Int. Telecomm. Union, no. G.992.2, 1999

[4] VDSLalliance, "SDMT VDSL draft standard proposal," Tech. Rep. T1E1.4/97-332, American National Standard for Telecomm., 1997.

[5] ETSI, "Digital video broadcasting (DVB); interaction channel for digital terrestrial television (DVBRCT)incorporating multiple access OFDM," Tech. Rep. EN 301958 DVB-RCT, ETSI, 2001.

[6] ETSI, "Broadband radio access networks (BRAN); HIPERLAN-2; physical layer," tech. rep., ETSI, 2000.

[7] N. Al-Dhahir and J. Cioffi, "Optimum finite length equalization for multicarrier transceivers," IEEE Trans. on Comm., vol. 44, pp. 56-64, Jan. 1996.

[8] G. Arslan, B. L. Evans, and S. Kiaei, "Equalization for discrete multitone transceivers to maximize bit rate," IEEE Trans. on Signal Proc., vol. 29, pp. 845-866, Dec. 2001.

[9] B. Farhang-Boroujeny and M. Ding, "An eigen-approach to design of near-optimum time domain equalizer for DMT tranceivers," in IEEE Int. Conf. on Comm., pp. 937-941, 1999.

[10] P. Melsa, R. Younce, and C. Rohrs, "Impulse response shortening for discrete multitone transceivers," IEEE Trans. on Comm., vol. 44, pp. 1662-1672, Dec. 1996.

[11] K. V. Acker, G. Leus, M. Moonen, O. van de Wiel, and T. Pollet, "Per tone equalization for DMT-based systems," IEEE Trans. on Comm., vol. 49, pp. 109-119, Jan. 2001.

[12] G. Goertzel, An Algorithm for the Evaluation of Finite Trigonometric Series, vol. 65, pp. 34-35. American Math. Monthly, 1958.

[13] A. Oppenheim and R. Schafer, Discrete-Time Signal Processing. Prentice-Hall Inc., 1992.

[14] M. Milosevic, L. F. C. Pessoa, B. L. Evans, and R. Baldick, "Optimal time domain equalization design for maximizing data rate of discrete multi-tone systems," IEEE Trans. on Signal Proc., Submitted Feb. 2002.

[15] J. Cioffi, "A multicarrier primer," Tech. Rep. T1E1.4/91157, Amati Comm. Corp. and Stanford University, 1991.

[16] J. Cioffi, G. Dudevoir, M. Eyuboglu, and G. D. Forney, "Minimum mean-square-error decision feedback equalization and coding - parts I and II," IEEE Trans. on Comm., vol. 43, pp. 2582-2604, Oct. 1995.

[17] J. Demmel, Applied Numerical Linear Algebra, pp. 173-176. SIAM, 1997.

[18] G. Arslan, M. Ding, B. Lu, M. Milosevic, Z. Shen, and B. L. Evans, "Matlab DMT TEQ design toolbox," July 2001. 